\title{
GENERALIZATION OF HILBERT'S INTEGRAL INEQUALITY
}

\author{
I. BRNETIĆ AND J. PEČARIĆ
}

Abstract. A generalization of the well-known Hilbert's inequality is given and several other results of this type obtained in the recent years follow as a special case from our result.

Mathematics subject classification (2000): 26D15.

Key words and phrases: Hilbert's inequality, Hölder's inequality, Beta function, Gamma function.

\section{REFERENCES}

[1] Bicheng YANG, On Hilbert's integral inequality, J. Math. Anal. Appl 220(1998) 778-785

[2] Bicheng YAng, On a General Hardy-Hilbert's Integral Inequality, Chin. Ann. of Math. 21A(2000) $401-408$

[3] BICHENG YANG, On anextension of Hardy-Hilbert's Integral Inequality, Chin. Ann. of Math. 23A:2(2002)

[4] T. C. PEACHEY, Some Integral Inequalities Related to Hilbert's, Journal of Inequalities in Pure and Applied Mathematics, 4(1), Art.19 (2003) 1-8

[5] IOAN GaVREA, Some Remarks On Hilbert's Integral Inequality, Mathematical Inequalities and Applications, 5(3)(2002) 473-477

[6] Yong Hong, All-sided generalization about Hardy-Hilbert integral inequalities, Acta Mathematica Sinica, 44:4(2001) 619-626 